\title{
The effect of scattering on the structure and SED of protoplanetary disks
}

\author{
C. P. Dullemond and A. Natta \\ 1 Max-Planck-Institut für Astrophysik, Postfach 1317, 85741 Garching, Germany \\ e-mail: dullemon@mpa-garching .mpg.de \\ 2 Osservatorio Astrofisico di Arcetri, Largo E. Fermi 5, 50125 Firenze, Italy
}

Received 30 January 2003 / Accepted 12 May 2003

\begin{abstract}
In this paper we investigate how the inclusion of scattering of the stellar radiation into a passive flaring disk model affects its structure and spectral energy distribution, and whether neglecting it could significantly decrease the model reliability. In order to address these questions we construct a detailed 1+1D vertical structure model in which the scattering properties of the dust can be varied. Models are presented with and without dust scattering, and for different albedos and phase functions. It is found that scattering has the effect of reducing the disk temperature at all heights, so that the disk "shrinks", i.e., the density at all intermediate heights decreases. However, this effect in most cases is more than compensated by the increase of the total extinction (absorption + scattering) cross section, so that the surface scale height increases, and images in scattered light will see a slightly thicker disk. The integrated infrared emission decreases as the albedo increases, because an increasing part of the flux captured by the disk is reflected away instead of absorbed and reprocessed. The reduction of the infrared thermal emission of the disk is stronger at short wavelengths (near infrared) and practically negligible at millimeter wavelengths. For relatively low albedo $(\omega \lesssim 0.5$ ), or for strongly forward-peaked scattering ( $g$ roughly $>0.8$ ), the infrared flux reduction is relatively small.
\end{abstract}

Key words. accretion, accretion disks - stars: circumstellar matter - stars: formation stars: pre-main-sequence - infrared: stars

\section{Introduction}

In recent years, models of irradiated passive protoplanetary disks have proven to be quite successful in explaining the spectral energy distributions (SEDs) of T Tauri (TTS) and Herbig Ae/Be (HAeBe) stars (Kenyon \& Hartmann 1987; Calvet et al. 1991, 1992; Malbet \& Bertout 1991; Chiang et al. 1997, 2001; D’Alessio et al. 1998, 1999; Malbet et al. 2001; Natta et al. 2001; Dullemond et al. 2001; Dominik et al. 2003). Though the exact geometry of such disks is still an issue of debate, the basic idea of a passively reprocessing dusty disk, possibly with a remnant tenuous envelope surrounding it, thusfar seems to withstand the test of time. However, it is clear that analyzing the SEDs alone is in most cases not sufficient to constrain the disk's parameters or to test theories (e.g. Chiang et al. 2001), and that spatially resolved images at various wavelengths are required in addition. Spatially-resolved images at millimeter wavelengths and optical and near-infrared images in scattered light provide valuable constraints to the disk geometry and to the properties of the dust in these disks (see, for example, D'Alessio et al. 2001; Testi et al. 2001, 2003 for the use of millimeter maps to constrain outer disk properties and Cotera et al. 2001 and references therein for the interpretation of disk images in scattered light). Models that self-consistently

Send offprint requests to: C. P. Dullemond, e-mail: dullemon@mpa-garching.mpg.de and simultaneously compute the disk structure, the SED and scattered-light images are required in order to fully exploit all the observed data. Some self-consistent models of this kind exist (e.g. D'Alessio et al. 2001; Malbet et al. 2001), but many other models use the assumption of zero albedo while computing the structure and SED of the disk (e.g. the models of Chiang et al. and those of Dullemond et al.). Most of the calculations of scattered light disk images use parametric disk models to describe the disk structure (see, for example, Wood et al. 1998, 2002; Wolf et al. 2003). Although very successful in deriving a first characterization of the disk surface properties, this approach does not take into account the effects of scattering on the structure and thermal emission of the disk.

In this paper, we explore systematically the effect on the disk structure and emission of including scattering of the stellar radiation by the grains in the disk. We do this by varying over a large range of values the albedo and phase function of the grains, and we consider disks heated by a typical Herbig Ae stars and by a typical TTS. Our study is somewhat complementary to D'Alessio et al. (2001), who present disk models where the grain size distribution is extended to very large grains and the cross sections for absorption and scattering are changed accordingly. Their approach is more physical, since it relates the adopted grain model to the specific physical process of grain growth. However, a parametric study like ours has the advantage of greater freedom in choosing the dust properties, and this 
is particularly important when one is interested in understanding the relevance of scattering in disk models in general.

In Sect. 2 we present a self-consistent 1+1D vertical structure model for an irradiated passive flaring disk around a TTS or a HAeBe star in which the scattering properties of the dust grains can be varied freely. In the case of zero albedo this model reduces to the earlier published model by Dullemond et al. (2002, henceforth DZN02). For non-zero albedo the differences to the latter model can be investigated in detail, and an assessment can be made of the role that scattering of the stellar radiation plays in the structure and SED of these disks. We illustrate the results for a disk annulus in Sect. 3, and for full disk models in Sect. 4. A summary and conclusions follow in Sect. 5.

\section{The vertical structure model with scattering}

The disk vertical structure model that we present in this section is based for most part on the 1+1D model of DZN02. The continuum radiative transfer, which is required in order to compute the dust temperature, is done in two stages. In stage 1, the primary stellar photons are followed as they travel from the stellar surface outwards into the surface layers of the flaring disk. In order to avoid having to solve a 2D problem, this is done using a "grazing incident angle recipe" (also called "flaring angle recipe") which splits the problem into a 1D vertical transfer problem at each radius. Photons are inserted into the disk at a flaring angle, which is computed self-consistently from the disk geometry. As the photons enter the surface layers, they get absorbed by the dust and leave their energy behind. In the second stage, the re-emission and subsequent radiative diffusion of this energy in the infrared regime is modeled by solving a 1D radiative transfer problem using the powerful variable Eddington factor technique. This technique allows us to solve the transfer problem at any optical depth, no matter how high. This is a major advantage over the often used (accelerated) lambda iteration techniques. It is assumed that the disk is passive, i.e. there is no internal production of heat by viscosity or any other mechanism. Therefore the above procedure suffices to determine the dust temperature at every height above the midplane. From this, the dust+gas density structure can be determined by integrating the equation of vertical hydrostatic equilibrium for a given surface density $\Sigma$, under the assumption that the gas and the dust temperatures are the same and the gas-to-dust ratio is constant (we take it 100). The entire procedure is iterated in order to get a self-consistent vertical temperature and density structure. It typically requires 4 to 6 iterations for a convergence to within $1 \%$. For more details on the model see DZN02.

In order to include scattering into this model, we replace stage 1 with a Monte Carlo code capable of modeling isotropic as well as small-angle scattering. In this way scattering of the primary stellar photons is included in a self-consistent way. The possible scattering of the re-emitted infrared photons can not be treated in this way, and will be ignored in the following. In practice, our code (and the results presented here) apply to disk atmospheres where the grains are relatively small, and their albedo is negligible at the wavelengths of the reprocessed radiation (i.e., in the infrared).
The flaring angle $\alpha$, under which the photons first enter the disk's surface layers before they scatter, is given by:

$\alpha=0.4 \frac{R_{*}}{R}+R \frac{\mathrm{d}}{\mathrm{d} R}\left(\frac{H_{\mathrm{s}}}{R}\right)$,

(see e.g. Chiang \& Goldreich 1997), where $H_{\mathrm{s}}(R)$ is the surface height of the disk, defined as the vertical height $Z$ at which the radial optical depth towards the star at stellar wavelength is unity. This flaring angle is recomputed after each iteration, so that in the end of the iteration procedure one obtains a selfconsistent solution with the proper flaring angle. As was described by Chiang et al. (2001), one has to employ a special method to compute the flaring angle such that numerical instabilities do not appear.

\subsection{Monte Carlo method}

In the Monte Carlo radiative transfer we let primary stellar photons impinge on the disk at an angle $\alpha$ with respect to the surface. We use a vertical 1D plane-parallel geometry, with a vertical spatial coordinate $Z$ ranging from $Z=0$ (the equator) to $Z=Z_{\max }$. In this approach, the incoming photons are assumed to have $\mu_{0}=-\sin (\alpha)$ in the traditional notation used in 1D radiative transfer. For each frequency bin we use $N$ photon packages. Each photon package is assumed to carry an energy (per unit disk surface area) of

$E_{v}^{0}=\frac{\alpha L_{v}^{*}}{4 \pi R^{2} N}$.

In the classical Monte Carlo approach each photon package is now followed as it scatters through the medium (randomly changing it's direction $\mu$ at each scattering event), until it gets absorbed at some point. Absorption is thus treated as a discrete event. By counting the number density of absorbed photon packages per grid cell, one can determine the energy deposition into each cell. This approach, although formally valid, has a serious weakness: it produces enormous numerical noise at small optical depths.

A better approach is to treat absorption as a continuous process, peeling off energy from the photon package according to the law:

$\frac{\mathrm{d} E_{v}}{\mathrm{~d} s}=-\rho \kappa_{v}^{\mathrm{a}} E_{v}$,

where $\kappa_{v}^{\mathrm{a}}$ is the absorption opacity, and $s$ is the path length along the photon trajectory which obeys the relation $\mathrm{d} s=\mathrm{d} z / \mu$. The energy lost by the package is deposited in the cell in which it was lost, while the package continues to move on until its energy $E$ has dropped below some prescribed fraction of the original energy $E_{v}^{0}$, or until the package has left the disk. Every time a photon package passes through a cell, another portion of energy is deposited into that cell, until all photon packages have been used up. The total energy (per unit surface area of the disk) deposited in the cell constitutes a heating rate $q$ per unit volume.

Scattering is still treated in a discrete way, and the entire package regularly changes direction due to these discrete scattering events. At the start of the random walk, and after each 
scattering event, a random number $\xi_{1}$ uniformly distributed in the domain $\langle 0,1\rangle$ is chosen using the random generator ran2 of Numerical Recipes (1992). Using this number, the location of the next scattering event is computed according to:

$z_{\text {new }}=z_{\text {current }}-\frac{\mu}{\rho \kappa_{\mathrm{S}}} \log \xi_{1}$.

At this location, the Henyey-Greenstein scattering function is invoked to compute the change in direction. This is first done in the photon's comoving frame. The Henyey-Greenstein (1941) function is:

$P\left(\cos \theta^{\prime}\right)=\frac{1}{2} \frac{1-g^{2}}{\left(1+g^{2}-2 g \cos \theta^{\prime}\right)^{3 / 2}}$,

where $g$ is the forward-peakedness parameter which is a property of the dust grains, and $\theta^{\prime}$ is the angle between the new photon direction and the old one. Using again the random number generator one can determine the $\theta^{\prime}$ :

$\cos \left(\theta^{\prime}\right)=\frac{1}{2 g}\left(1+g^{2}-\frac{\left(1-g^{2}\right)^{2}}{\left(1-g+2 g \xi_{2}\right)^{2}}\right)$,

where $\xi_{2}$ is again the random number between 0 and 1 . The other angle $\left(\phi^{\prime}\right)$ is simply a random number between 0 and $2 \pi$ : $\phi^{\prime}=2 \pi \xi_{3}$. We now employ a rotation matrix operation to rotate these angles to the real ones $\theta, \phi$, the new $\mu$ being $\mu=\cos \theta$.

Using the above procedure, one can compute the heating $q(z)$ due to the incidence of primary stellar photons. This heating rate then serves as the source term for stage 2 : the radiative transfer of thermal emission from the disk. The continuous treatment of absorption in the Monte Carlo simulation assures that this function is virtually free of numerical noise even for a modest number of photon packages (say, $N=100$ ). This method (which is similar to the method of van Zadelhoff et al. 2003) works very well at all optical depths. We have tested that the method reproduces the results of a Monte Carlo code using discrete absorption events.

\subsection{Comparing to diffuse reflection theory of Chandrasekhar}

The above numerical procedure can be tested against the analytic solutions of diffuse scattering (Chandrasekhar 1950/1960). Using so called H-functions, the problem of photons scattering off a semi-infinite slab with a certain albedo $\omega \equiv \kappa_{\text {scat }} /\left(\kappa_{\text {abs }}+\kappa_{\text {scat }}\right)$ can be reduced to a single numerical integral. For the case of isotropic scattering the $H$-function of Chandrasekhar is defined in the following way:

$\frac{1}{H(\mu)}=\sqrt{1-\omega}+\frac{\omega}{2} \int_{0}^{1} \frac{\mu^{\prime}}{\mu+\mu^{\prime}} H\left(\mu^{\prime}\right) \mathrm{d} \mu^{\prime}$.

The values of the $H$-function can be quickly found by iteratively evaluating Eq. (7) until concergence is reached. Tabulated values can be found in Chandrasekhar (1950/1960). Once this $H$-function is found, one can find the reflected fraction of incident flux $\eta$ as:

$\eta=\frac{1}{2} \omega \int_{0}^{1} \frac{\mu^{\prime}}{\mu_{0}+\mu^{\prime}} H\left(\mu_{0}\right) H\left(\mu^{\prime}\right) \mathrm{d} \mu^{\prime}$,

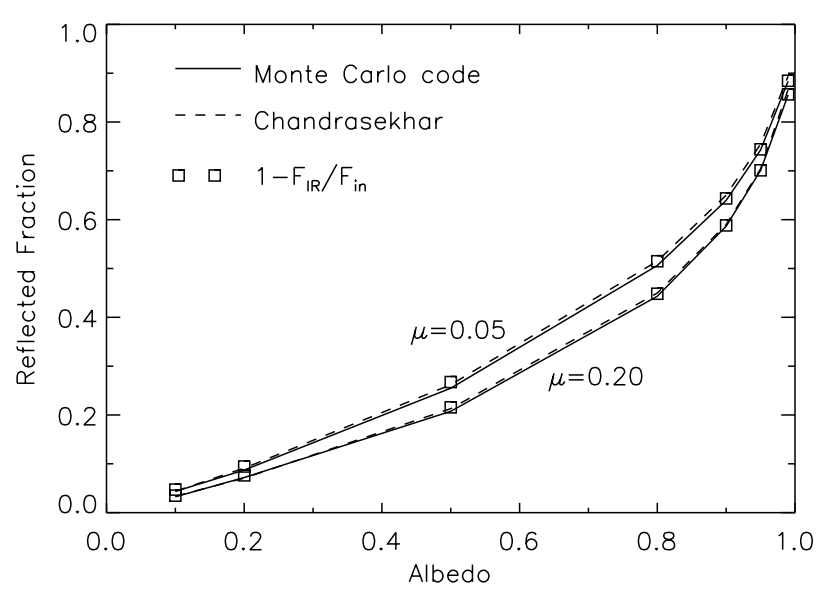

Fig. 1. The fraction of light diffusely reflected off a semi-infinite slab as a function of albedo of the slab. Compared are the analytic results of Chandrasekhar, the results from our Monte Carlo code, and the results from the thermal reprocessing computed by our code.

where $\mu_{0}$ is the incident angle of incoming flux, assuming that the flux is a parallel beam. In the case of a disk irradiated by a star, this assumption is reasonably well justified. The reflection fraction $\eta$ is a number between 0 and 1 , where $\eta=1$ means that every photons is eventually scattered away without having been absorbed, and $\eta=0$ means that all photons have been absorbed. In Fig. 1 the reflected fraction of incident flux is shown as a function of albedo. Plotted over each other are the predictions from Chandrasekhar's theory of $\mathrm{H}$-functions, the reflected fraction computed using our Monte-Carlo code, and one minus the fraction of the flux that is reprocessed into the infrared according to our code. All fall reasonably well over each other, so we trust our numerical results.

Figure 1 shows that for small grazing angle and small albedo one has $\eta \simeq \omega / 2$, meaning that the infrared luminosity of the disk is easily estimated to be $L_{\mathrm{IR}} \simeq(1-\omega / 2) L_{*}$. This result can be understood in terms of the Chiang \& Goldreich picture of a hot surface layer: this layer intercepts all stellar radiation, and redirects half of it away from the disk and half towards the disk. This redirecting proceeds partly through scattering $(\omega)$, partly through absorption $(1-\omega)$. This means that a fraction of $\omega / 2$ of the incident flux is scattered away from the disk surface. The other $\omega / 2$, which is directed towards the disk interior, for low albedo will be almost entirely absorbed, so that $(1-\omega / 2)$ will be reprocessed in the infrared. For larger albedo, the downward directed scattered photons might be scattered once more, and escape after all. This causes the deviation from the $\eta \simeq \omega / 2$ estimate, as seen in Fig. 1 .

\subsection{Approximate treatment of isotropic scattering}

For isotropic scattering phase function there is an interesting approximate alternative to the full Monte Carlo approach described above (Calvet et al. 1991; Strittmatter et al.1974). Using the moment equations of radiative transfer in the Eddington approximation one can derive an analytic formula for the mean intensity of the direct + scattered (but not the re-emitted) photon field $J_{v}$ as a function of vertical 
absorption + scattering optical depth into the disk $\tau_{v}$. If we define $\beta_{v}=\sqrt{3\left(1-\omega_{v}\right)}$, and $\mu_{0}=\sin (\alpha)$, where $\alpha$ is the grazing angle of incidence of the impinging stellar photons, then the mean intensity $J_{v}\left(\tau_{v}\right)$ can be written as:

$$
\begin{aligned}
J_{v}\left(\tau_{v}\right)= & \frac{\mu_{0} \omega\left(2+3 \mu_{0}\right) F_{v}^{*}}{4 \pi\left(1+2 \beta_{v} / 3\right)\left(1-\beta_{v}^{2} \mu_{0}^{2}\right)} \mathrm{e}^{-\beta_{v} \tau_{v}} \\
& -\frac{3 \mu_{0}^{2} \omega F_{v}^{*}}{4 \pi\left(1-\beta_{v}^{2} \mu_{0}^{2}\right)} \mathrm{e}^{-\tau_{v} / \mu_{0}}+\frac{F_{v}^{*}}{4 \pi} \mathrm{e}^{-\tau_{v} / \mu_{0}}
\end{aligned}
$$

(Calvet et al. 1991) where $F_{v}^{*}$ is the unextincted stellar flux at the radius of the disk annulus. Using this expression for the mean intensity of scattered + direct stellar radiation the heating rate $q(z)$ can be determined:

$q(z)=4 \pi \int_{0}^{\infty} J_{\nu}(z) \rho \kappa_{\nu} \mathrm{d} \nu$.

This $q(z)$ then serves as the source term to stage 2 of the radiative transfer calculation.

We have compared the models resulting from these equations to those resulting from the more precise Monte Carlo method described in Sect. 2.1. We found that under most circumstances, for isotropic scattering phase function, the temperature structure derived from the use of Eqs. (9), (10) and that from the Monte Carlo method differ by less than 3\%. For isotropic scattering, Eq. (9) therefore seems to be a reliable formula. However, it works only when the disk has large optical depth at all wavelength where the stellar flux is important. Also, it is important to note that the equation for the dust temperature proposed in Calvet et al. (their Eq. (14)) is not very accurate, as it is based on the use of the Rosseland mean opacity. As was discussed by DZN02, such an approach may lead to a wrong temperature structure.

\section{Single annulus setup}

In order to get an understanding of the effect of scattering on the disk structure we start with a single-annulus setup. The central star is assumed to have the following parameters: $M_{*}=2 M_{\odot}, R_{*}=2 R_{\odot}, T_{*}=10000 \mathrm{~K}$ (i.e. $L_{*}=36 L_{\odot}$ ). These are parameters relevant to Herbig Ae stars. For simplicity we assume the spectrum of the star to be a blackbody spectrum. The annulus of the flaring disk we wish to study is located at $1 \mathrm{AU}$, and has a width of $0.01 \mathrm{AU}$ (i.e. it lies between 1.00 and 1.01 AU from the central star). The grazing angle (i.e. the angle at which the stellar radiation enters the disk's atmosphere) is generally computed self-consistently from the disk model, but for the simple test case described here we assume it to be fixed to $\alpha=0.05$. The opacity is taken to be that of a silicate grain of $0.1 \mu \mathrm{m}$ (Draine \& Lee 1984). The scattering opacity is constructed artificially, in order to be able to investigate different values of the albedo. We assume that the scattering is zero for $\lambda \geq 2 \mu \mathrm{m}$, and that it is a fixed fraction of the absorption for $\lambda<2 \mu$ m (keeping the absorption opacity itself unchanged). In this way we can freely vary the albedo for the incident stellar radiation, while the albedo for the reprocessed radiation remains zero. It should be noted that with this definition the total (scattering+absorption) opacity for

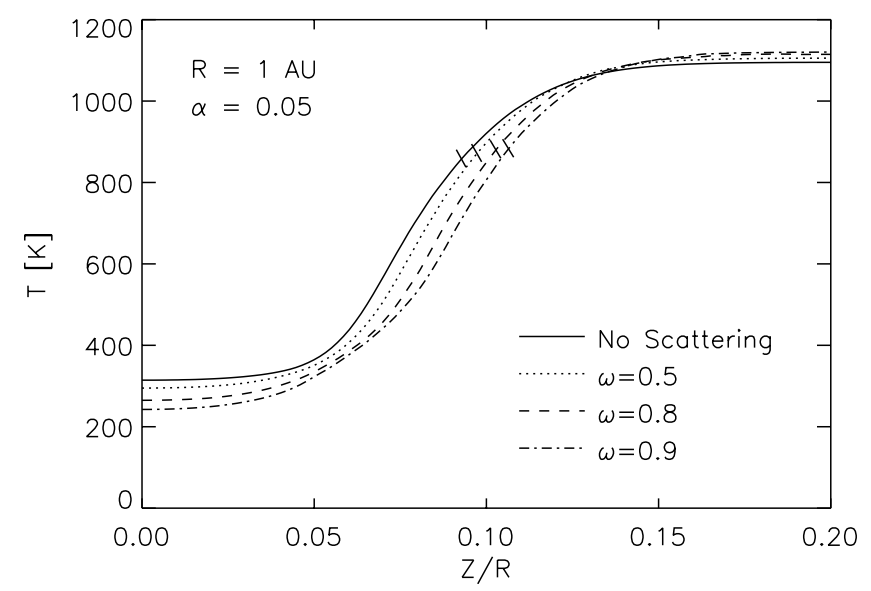

Fig. 2. Vertical temperature profile of the test annulus as function of $Z / R$ for different values of the albedo, as labelled. The tickmark on each curve indicates the position of the surface height $H_{\mathrm{s}}$ of the disk, i.e. the height where the optical depth (absorption + scattering) with respect to stellar photons is unity.

$\lambda<2 \mu \mathrm{m}$ increases for increasing albedo. The surface density of our test setup is defined using the optical depth for the case of zero albedo. The annulus has a surface density of $\Sigma=0.3$, and (for $\omega=0$ ) a visual optical depth in the vertical direction of $\tau_{\mathrm{V}}=10$.

For the setup described above we compute a number of models varying the albedo and the phase angle. In a first set of models we assume that the scattering is isotropic and change the albedo in the range $0.5-0.9$. In a second set of models, we will fix the albedo to be $\omega=0.8$ and consider forward-peaked scattering by varying the phase angle from 0.0 to values in the interval 0.5-0.9. In the ISM, typical values of the albedo are of about 0.5, with $g \sim 0.5$ (Draine \& Lee 1984; Kim et al. 1994). Both parameters, however, depend strongly on the grain properties, such as size and chemical composition (e.g. Mishchenko et al. 2000).

\subsection{Varying the albedo}

In Fig. 2 the vertical temperature profile is shown for four values of the albedo, from 0.0 to 0.9 and isotropic scattering. As the albedo increases, an increasing fraction of the stellar energy is lost from the heating budget. The effect of scattering is to decrease the disk temperature at all heights, with the only exception of the very optically thin region on the surface, where the stellar photons reflected away from the disk slightly increase the mean intensity of the heating radiation. The temperature decrease is larger at intermediate optical depth, while the midplane temperature drops only slightly, by $6 \%$ for $\omega=0.5$ and a maximum of $23 \%$ for $\omega=0.9$. It is easy to understand why this effect is so small if one looks at it from a two-layer perspective (Chiang \& Goldreich 1997). The disk midplane is heated by the emission from the surface layer, part of which is thermal dust continuum $(1-\omega)$ and part of it is scattered light $(\omega)$. Only a fraction $(\omega / 2)$ of the downward scattered light has a chance to scatter backwards away from the disk, and escape the fate of being absorbed and reprocessed. This means that the effect 
on the midplane temperature should be of the order of $\omega^{2}$ (see Sect. 2.2).

Figure 3 shows the corresponding density profiles. One can see the deviation from the Gaussian vertical profile expected in an isothermal disk. The effect is significant at high $Z$, where the increase of the temperature due to stellar irradiation occurs. One can also see that at fixed $Z$ the density is lower for higher values of $\omega$. However, the effect of scattering on the pressure scale height $H_{\mathrm{p}}$ is minimal, since $H_{\mathrm{p}}$ depends on the square root of the midplane temperature, which changes very little (see Fig. 2).

Figures 2 and 3 show the location of the surface height $H_{\mathrm{s}}$, i.e., where the stellar-flux-averaged optical depth (absorption + scattering) to the stellar photons entering the disk under the angle $\alpha$ is unity. The location of $H_{\mathrm{s}}$, which defines the "surface" of the disk, moves upwards as $\omega$ increases, reflecting the increase in total optical depth as more scattering opacity is added. Even if for increasing albedo the density structure of the disk "shrinks", this effect is counteracted by the increased total opacity, and $H_{\mathrm{s}}$ in fact moves towards higher $Z / R$.

It is interesting to note that intuitively one might expect scattering to increase the temperature of the disk just below the surface $H_{\mathrm{s}}$, since scattering redirects stellar radiation deeper into the disk. However, as can be seen in Fig. 2, such an increase in temperature does not take place. The explanation lies in energy conservation. The deep layers of the disk get heated indirectly via emission from the surface layer of the disk. Without scattering this downward flux is purely thermal emission from the hot dust in the surface layer. When scattering is included, the total flux that is beamed downward stays the same, but now consists partly of thermal emission from the surface layer and partly of scattered light at stellar wavelengths. The only effect that can be discerned is that, due to the different absorption cross sections in these two wavelength ranges, the scattered photons are absorbed higher up in the disk than the thermal photons. This very subtle effect is seen in the $\omega=0.9$ temperature curve in Fig. 2 between $Z / R=0.04$ and $Z / R=0.08$, where the slope changes less rapidly than for smaller values of $\omega$.

Figure 4 shows the resulting SEDs for our test annulus for the same values of the albedo $\omega=0,0.5,0.8,0.9$. Only the thermal emission from the annulus is shown, not the scattered-away radiation which remains at stellar wavelengths. As one can see, the main effect is to reduce the overall infrared flux from the annulus. This is a logical consequence of energy conservation: as more radiation is scattered away instead of being absorbedand-re-emitted, the total budget of reprocessable radiation is of course lower. In this particular example, the fraction of reprocessed (i.e. not scattered) stellar radiation varies from 1 for $\omega=0$ to 0.75 for $\omega=0.5$ and to 0.37 for $\omega=0.9$.

At short wavelengths the reduction is stronger than at long wavelengths, so that the SED appears redder. At mm wavelengths the reduction between $\omega=0$ and $\omega=0.5$ is about $6 \%$ (proportional to the decrease of the midplane temperature), while at $\lambda=3 \mu \mathrm{m}$ the reduction is about $40 \%$. If one quantifies the reddening by the ratio of the NIR/IR emitted luminosity, with NIR between 1 and $7 \mu \mathrm{m}$, it reduces from 0.19 for $\omega=0$, to 0.16 for $\omega=0.5$ and 0.09 for $\omega=0.9$.

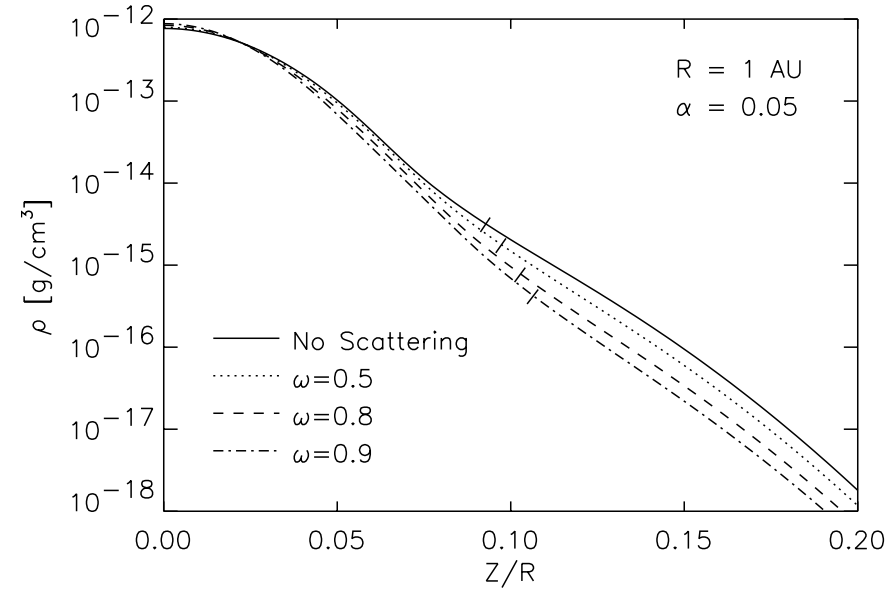

Fig. 3. Same as Fig. 2 but now for the vertical density profile.

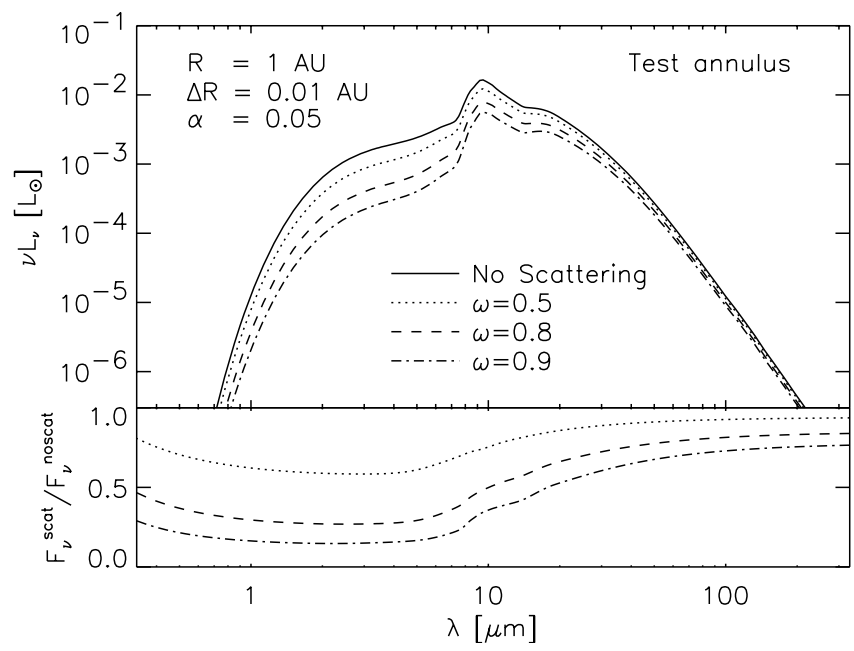

Fig. 4. SED of the test annulus for different values of the albedo, as labelled. The flux from the star is not included, nor is the flux from the radiation that is scattered away from the disk. Only the thermal emission is shown.

The reason for this is that the NIR emission forms in the upper layers of the disk where scattering has a stronger effect than near the midplane. At very long long wavelengths the disk emission is proportional to the temperature of the midplane, which, as we have discussed, decreases only little.

\subsection{Small angle scattering versus isotropic scattering}

In order to find out which effect a non-isotropic scattering phase function has on the structure of the disk and on the SED, we take the single-slice setup of the previous section, and make runs for different values of $g$, using the Henyey-Greenstein phase function. For $\alpha=0.05$ and $\omega=0.8$ the differences in the $\mathrm{SED}$ are shown in Fig. 5. From this figure it can be seen that increasing $g$ has the effect of decreasing the influence of scattering on the disk. As the $g$ factor increases, the infrared spectrum of the annulus also increases, since the scattering becomes less effective in scattering radiation away before it can thermalize and be reprocessed into the infrared. For increasing $g$, the SED 


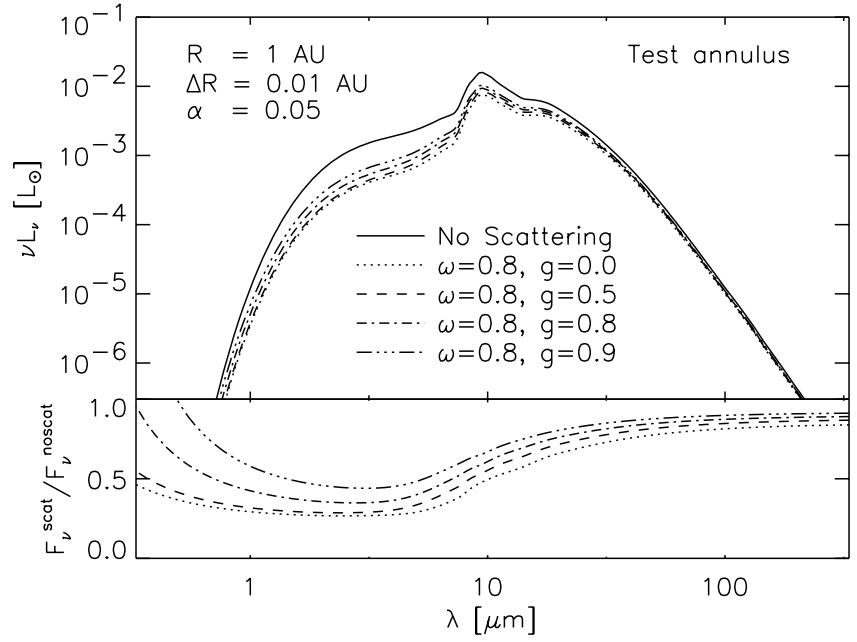

Fig. 5. The effect of increasing the small-angle scattering factor $g$ for constant albedo $\omega$ on the spectrum of the annulus test case.

progressively moves towards the SED as computed from the case without scattering.

The reason why this is the case can be understood by taking the extreme example of perfectly forwardly peaked scattering $(g=1)$, which is equivalent to no scattering at all, since a scattered photon will keep moving in the same direction as before the scattering event. For $g=1$, one can therefore be sure that the results should be identical to the case of no scattering, even for high albedo. For smaller $g$ scattering becomes important, but the forward peakedness of scattering will supress the effect of scattering compared to the case of $g=0$. This is because the forward peakedness of scattering phase function improves the chance of still pointing into the disk after the first scattering event, but still leaves a chance to get scattered away.

The question now arises: could one simulate the effects of small angle scattering by isotropic scattering with a reduced "effective" albedo? To answer this we set up the following procedure. We start with a calculation of the kind described above with a particular $\omega$ and $g$. Then we make a new calculation with $g=0$, and tune the $\omega$ until we obtain the same reflection fraction as in the original computation. This "effective" albedo $\omega_{\text {eff }}$ can be automatically found by placing the model computation as a function-call inside a root finding routine (for instance zbrent from Numerical Recipes 1992). We found that for any value of the parameters $R, \alpha, \omega, g$ we explored, it is possible to find a value of $\omega$ (called $\omega_{\text {eff }}$ ) that reproduces the same SED with $g=0$ to within 5\%. This effective albedo is always $\omega_{\text {eff }} \leq \omega$. In Fig. 6 the effective reduction of the albedo (i.e. $\omega_{\text {eff }} / \omega$ ) is shown for increasing value of $g$ for different values of the incident grazing angle $\alpha$ and original albedo $\omega$. One can see that for small $\omega$ the reduction is stronger than for big $\omega$. This can be explained by the fact that high albedo gives a photon more than one chance to scatter and change its direction. Multiple scattering will erase the memory of the original direction where the photon came from. One also sees from the figure that a higher incident angle $\alpha$ will aggravate the reduction effect. This is because for higher $\alpha$ the photon will need a larger scattering angle in order to be able to escape the disk.

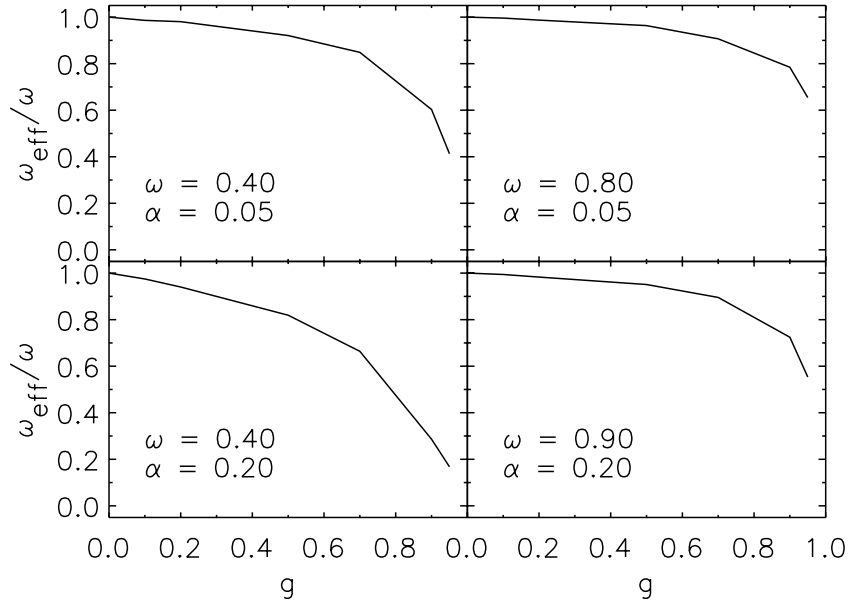

Fig. 6. The effective reduction of the albedo as a result of nonisotropic scattering, for different values of $\omega$ and incident grazing angle $\alpha$.

In the extreme case of $\alpha=\pi / 2$ (vertical incident radiation) a photon will need to scatter essentially backwards in order not to get absorbed within the disk. For this case the counter effect of $g$ will be the strongest. For the limiting case of $\alpha=0$, small angle scattering will essentially change nothing, since even a small scattering angle has $50 \%$ chance of deflecting the photon away from the disk.

An analytic fitting formula for the effective albedo is:

$\omega_{\mathrm{eff}}=\omega\left(1-g^{a}\right)^{b}$

with

$a=3.7 \omega^{2.9}-1.6 \alpha^{0.42}+2.5$

$b=-0.31 \omega^{2.9}+1.3 \alpha^{0.42}$.

This formula has a mean deviation of $10 \%$ with the computed values within the domain of $0 \leq g \leq 1,0.01 \leq \alpha \leq 1$ and $0.1 \leq \omega \leq 0.9$.

\section{Full disk models}

We turn now to the determination of the effects on the SED of a full disk ranging from the inner dust-evaporation radius out to a few hundred AU. We have set up a disk model for the same Herbig Ae star parameters as in Sect. 3: mass $M_{*}=2 M_{\odot}$, effective temperature $T_{*}=10000 \mathrm{~K}$ and luminosity $L_{*}=36 L_{\odot}$. The disk surface density is taken to obey $\Sigma(R)=400(R / \mathrm{AU})^{-1} \mathrm{~g} / \mathrm{cm}^{2}$ with an inner radius of $1.0 \mathrm{AU}$ and outer radius $300 \mathrm{AU}$. The mass of this disk is $M_{\text {disk }}=0.08 M_{\odot}$. In these models the flaring angle and pressure scale height are computed self-consistently, as in DZN01. We vary the albedo from $\omega=0$ to $\omega=0.9$ and assume isotropic scattering. We ignore, for the moment, the emission from the inner rim of the disk.

The resulting SEDs are shown in Fig. 7. As expected following the discussion in Sect. 3, scattering has the effect of reducing the infrared excess over the entire wavelength range. The ratio of the reprocessed luminosity to the stellar one varies from 0.41 for $\omega=0$ to 0.36 for $\omega=0.5$ to 0.19 for $\omega=0.9$. 


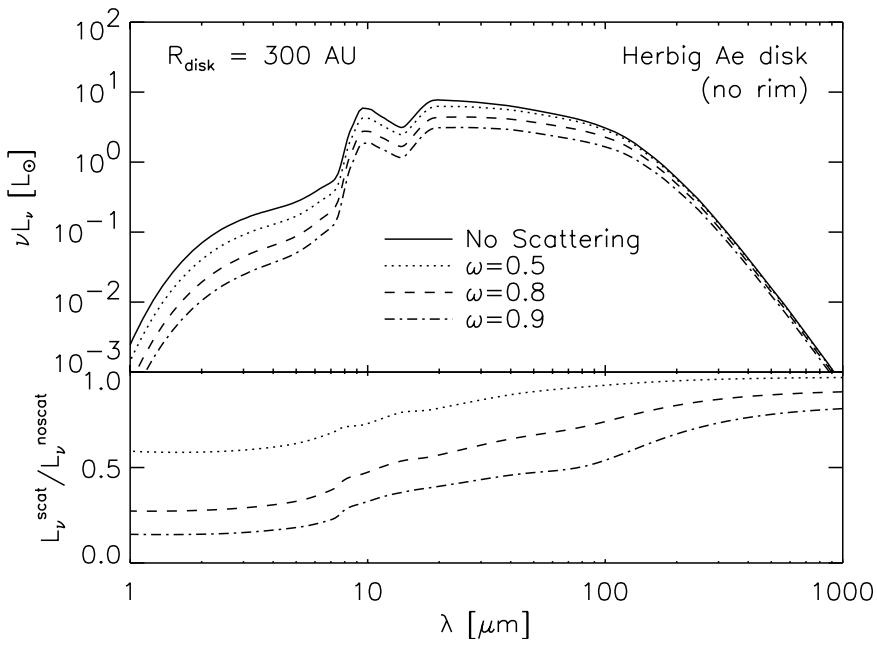

Fig. 7. The effect of increasing albedo on the total SED of a passive flaring irradiated disk around a Herbig Ae star. The SED is computed at an inclination of $i=45^{\circ}$. No inner rim is included. Only the thermal emission from the disk is shown.

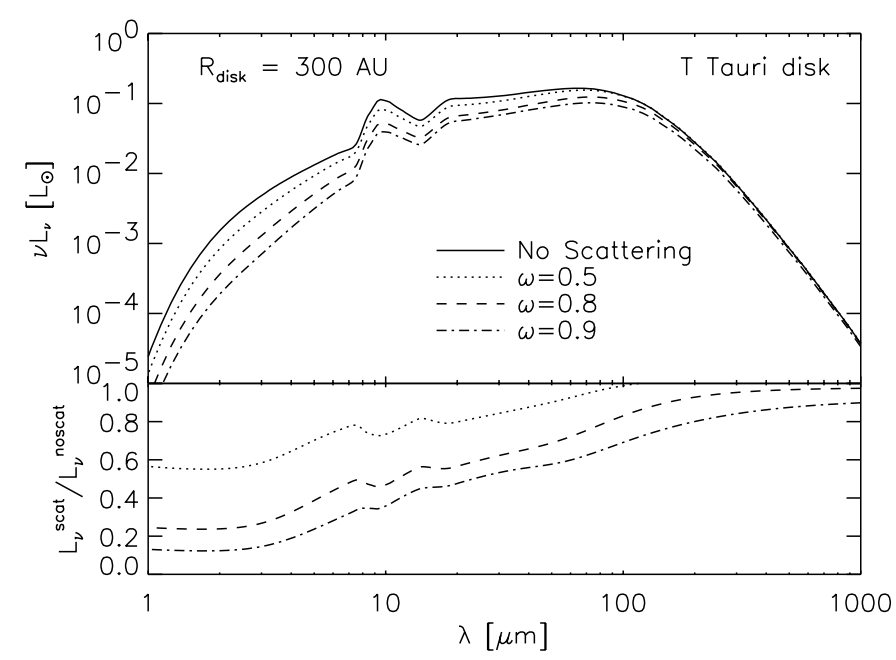

Fig. 8. Same as Fig. 7 for a TTS.

Roughly speaking, the effect of scattering on the infrared excess is to reduce it by a factor of the order of (1- $\omega / 2)$. This is not exact (see Sect. 2.2), but gives a zero order of magnitude of the effect.

As in the case of the single annulus setup the reduction is stronger at short wavelengths than at long wavelengths. This is more clearly seen in the lower panel of Fig. 7, in which the reduction factor is plotted as a function of wavelengths. The reduction is practically negligible at millimeter wavelengths, while it is as large as $80 \%$ (for $\omega=0.9$ ) in the near infrared. It is interesting to note that the reduction factor is a rather smooth function of wavelength, and that it shows only a weak variation around the location of the $10 \mu \mathrm{m}$ silicate feature. The degree by which one sees this effect varies from model to model. It is a subtle effect of the precise vertical profile of the temperature and the density in the disk, and is also linked to the slight reduction of the disk's interior temperature for increasing albedo. If we enforce the midplane temperature of the disk to be unaffected by the scattering (by fixing the temperature at $z=0$

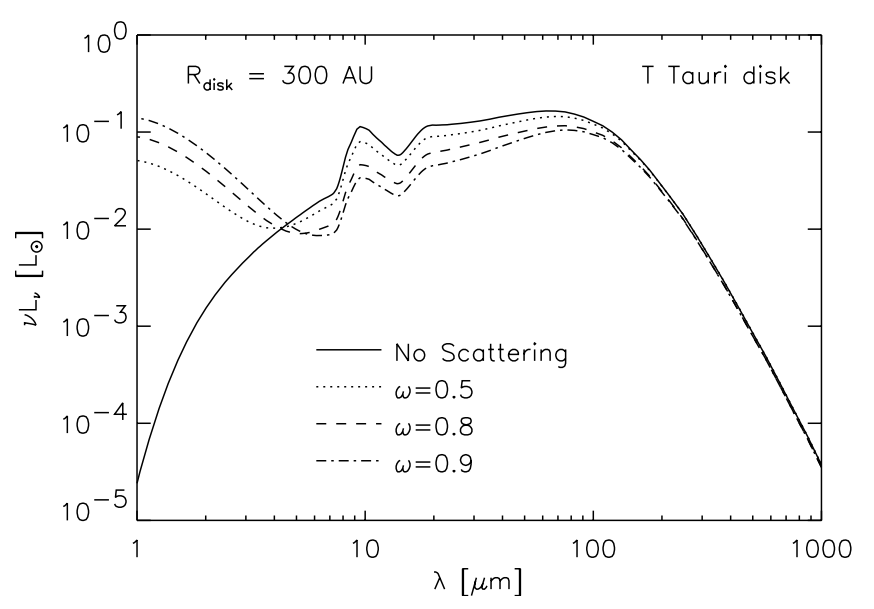

Fig. 9. Same as Fig. 8, but now with the scattered light from the disk included. The direct light from the star is not included.

to the value it had without scattering), then the $10 \mu \mathrm{m}$ feature turns up quite strongly in the reduction factor.

Figure 8 shows the SEDs of disks irradiated by a T Tauri star (TTS). The parameters of the star are taken to be: $T_{*}=4000 \mathrm{~K}, R_{*}=2 R_{\odot}, M_{*}=0.5 M_{\odot}$. The disk has an outer radius of $300 \mathrm{AU}$, an inner radius of $R_{\text {in }}=0.1 \mathrm{AU}$, a mass of $M_{\text {disk }}=0.008 M_{\odot}$, and a powerlaw index of the surface density distribution of -1 . The results are qualitatively very similar to the Herbig Ae star case. For $\omega=0.5$, however, an interesting phenomenon occurs. Longwards of $100 \mu \mathrm{m}$ the reduction factor for the SED exceeds unity, i.e. an inflation instead of reduction. This can be understood as a result of the increasing height $H_{\mathrm{s}}$ of the disk, as can be seen in Fig. 10. The grazing angle $\alpha$ increases, enhancing the irradiation. This effect however is rather small, and on the whole the infrared excess is reduced by the inclusion of scattering.

In Fig. 9 the same SEDs is shown as in Fig. 8, but this time the scattered light from the disk is included in the spectrum (the direct stellar light is still not included). As one can see: the flux of the scattered light increases for increasing albedo. This is a combined effect of the disk becoming more refractory and the increasing height $H_{\mathrm{s}}$.

In addition to the surface height $H_{\mathrm{s}}$, Fig. 10 also shows the pressure scale height at the equatorial plane $H_{\mathrm{p}}$. Contrary to $H_{\mathrm{s}}$, the pressure scale height varies only little as a function of albedo.

\subsection{Inner rim}

For Herbig Ae/Be stars there is evidence that, in computing the SED, one should include the effect of the stellar radiation impinging frontally on the disk at the dust sublimation radius (Natta et al. 2001; Dullemond et al. 2001 henceforth DDN01). Since scattering of the stellar light in this inner rim may be important, we have computed the SED of rim+disk models for various values of the albedo. The rim is treated in a way similar to that of DDN01. Knowing that the rim is located at the dust evaporation radius, we know its temperature (about $1500 \mathrm{~K}$ ) and we can compute the pressure scale height of the rim. The surface height of the rim (the $z$ above the midplane at which the 


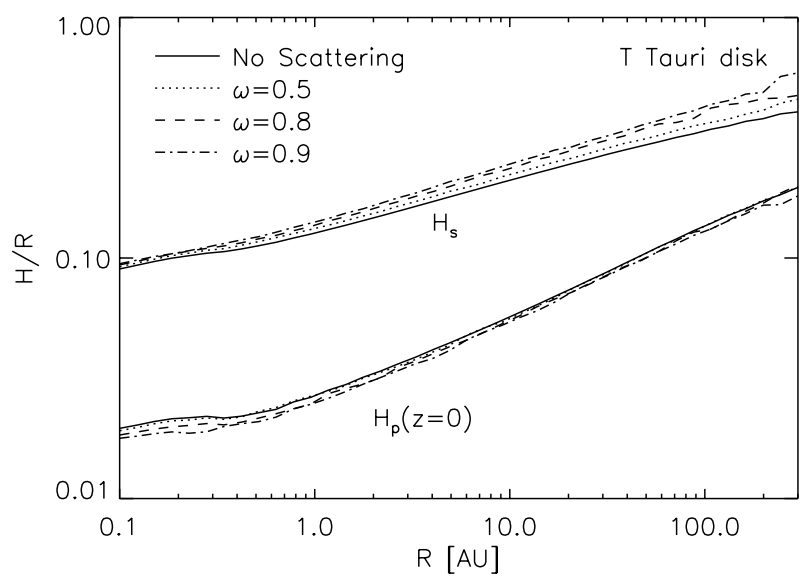

Fig. 10. Run of the pressure scale height $H_{\mathrm{p}}$ and of the surface scale height $H_{\mathrm{s}}$ as function of $R$ for different values of the albedo, as labelled.

rim becomes transparent to starlight) is then easily computed. As in DDN01, this height is considerably higher than the nominal height of the flaring disk at that radius, so this rim will in fact cast a shadow over the flaring disk. The SED from this disk consists then of a component in the near infrared originating from the hot inner rim, and the emission in mid- and farinfrared originating from the non-shadowed part of the flaring disk behind the rim. Scattering is included in the treatment of the inner rim using the same 1D plan-parallel radiative transfer techniques used for the disk annuli, but this time in the horizontal direction.

The results are shown in Fig. 11. The SED is very similar to that of models with no rim at long wavelengths (in this example approximately for $\lambda \geq 15 \mu \mathrm{m}$ ), where the emission comes from regions of the disk which are out of the rim shade. The region shorter of about $7 \mu \mathrm{m}$ is deeply affected by the presence of the rim, which reprocesses in this region the intercepted stellar radiation. The effect on the inner rim emission of increasing $\omega$ can be seen clearly in Fig. 11, lower panel, and it is qualitatively similar to what we have discussed in Sect. 3: the emission decreases at all wavelengths, with the exception of the very short ones, where it is fixed by the requirement that $T=1500 \mathrm{~K}$. Globally, however, the overall shape of the SED depends on $\omega$ less than that of models with no rim and, in particular, the nearIR excess $(\lambda<7 \mu \mathrm{m})$ is always roughly $25 \%$ of the total. The total infrared excess of these models is always higher than in models with no rim. However, it decreases in a similar way with $\omega$, roughly as $(1-\omega / 2$ ) (from 0.61 to 0.50 for $\omega=0.5$ and to 0.32 for $\omega=0.9$ ).

We also made a set of rim+disk models for fixed albedo $(\omega=0.5)$, but varying $g$ between isotropic scattering and strongly forward-peaked scattering (see Fig. 12). Increasing the value of $g$ decreases the effects of scattering on the SED, but it does so more effectively for the inner rim (near-IR) than for the flaring disk (mid-far-IR). The reason is that the inner rim is irradiated under an incident angle of $i=90^{\circ}$, while the flaring disk is irradiated under a small incident angle. For small incident angles a photon is still likely to skim off the surface even for large $g$, which is not the case for large incident angle (see Sect. 3.2).

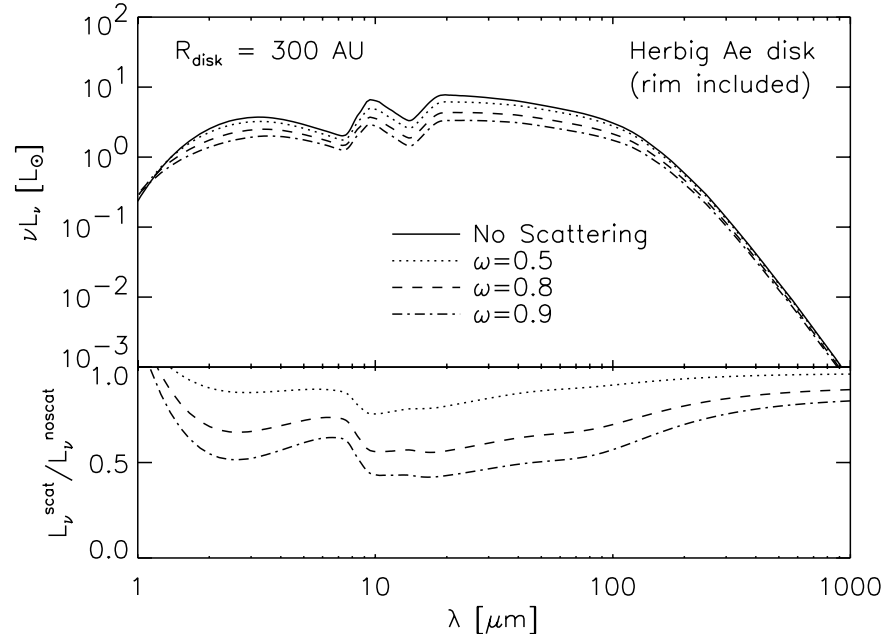

Fig. 11. Same of Fig. 7, but with the inner rim included.

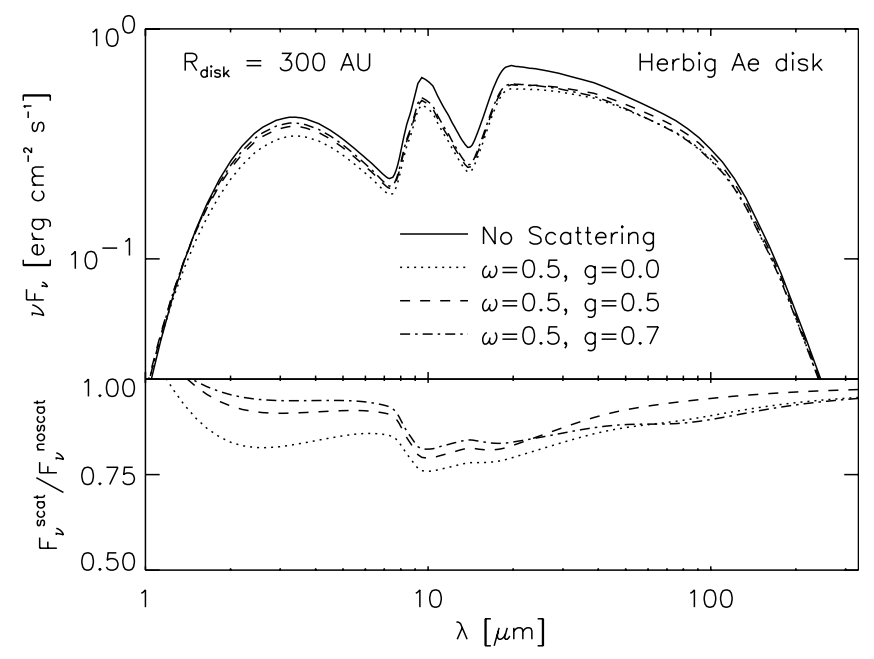

Fig. 12. The effect of varying $g$ on the SED of a passive flaring irradiated disk around a Herbig Ae star. In all cases it is $\omega=0.5$. These models include the inner rim. Note that the vertical scales are different from previous figures, in order to show the effects more clearly.

\section{Summary and conclusion}

In this paper we investigate the effects of scattering of the impinging stellar radiation on the structure and spectral energy distribution of a passive flaring irradiated circumstellar disk. We base our model calculations on the detailed vertical structure model of DZN02, in which full-fledged radiative transfer is used on a density structure in vertical hydrostatic equilibrium. It was shown in that paper that the inclusion of full frequencyand-angle dependent radiative transfer (although in a $1+1 \mathrm{D}$ manner) can have profound effects on the vertical structure, most notably on the dust temperature at the equatorial plane. Here we improve these models by replacing the original treatment of irradiation by a Monte Carlo approach that can handle small-angle scattering by dust particles ${ }^{1}$.

We compared models without scattering to models with increasing albedo at the wavelengths of the stellar radiation.

\footnotetext{
1 All the models presented in this paper can be downloaded in numerical form from http://www.mpa-garching.mpg.de/ PUBLICATIONS/DATA/radtrans/diskscat/.
} 
Note that we keep the absorption cross section fixed, so that increasing albedo results in increasing the total optical depth (scattering + absorption) for the stellar radiation. As the albedo increases, the disk gets cooler at all vertical heights, with the only exception being the very upper layers. This reduction of the temperature takes place even at the very optically thick midplane, although the effect is smaller than at intermediate optical depth. As a result, the disk becomes slightly flatter (i.e. it "shrinks" in vertical direction). Still, the surface scale height $H_{\mathrm{s}}$, which defines the surface where the stellar light is intercepted, increases, since the increase of the total opacity more than compensates for the physical disk shrinking. Disks seen in scattered light will appear thicker than predicted by models where scattering is ignored.

As far as the SED is concerned, increasing the albedo has the effect of reducing the thermal emission from the disk over the entire infrared wavelength domain. This is because scattering reflects part of the stellar light away from the disk before it had a change to get absorbed and reprocessed into the infrared. To zero order, for $\omega<0.5$ the reduction is approximately $(1-\omega / 2)$, and somewhat larger for larger values of the albedo. However, we find that the spectrum is more strongly affected at short than at long wavelengths, so that the overall SED looks "redder". For a typical case with $\omega=0.5$ the reduction in the near and mid-IR could be as large as $40 \%$, while at $\mathrm{mm}$ wavelength the reduction is of few percent at most. Models computed with different values of the phase function show that the effect of scattering decreases strongly when it is more forward-peaked.

In summary, we conclude that the effect of scattering of the stellar radiation by grains in the disk has to be considered carefully in disk models, unless the albedo is low $(\omega \ll 0.5)$, the scattering is extremely forward-peaked, or the required accuracy is not very high. Including scattering self-consistently in the determination of the disk's structure is particularly important when one is interested in predicting the disk shape that one sees in scattered light, and/or the near and mid-infrared emission. Only at very long wavelengths the disk properties (both integrated flux and intensity profile) are practically unaffected by the fraction of stellar light that is scattered rather than absorbed by the disk.

Acknowledgements. We thank the anonymous referee for many useful comments and suggestions which have improved the paper considerably. We wish to thank E. Krügel for kindly lending us his 1D planeparallel radiative transfer code which we used to test our own 1+1D disk structure code. We also thank G. J. van Zadelhoff for discussions that proved to be useful during the development of our Monte Carlo code. CPD acknowledges support from the European Commission under TMR grant ERBFMRX-CT98-0195 (“Accretion onto black holes, compact objects and prototars").

\section{References}

Calvet, N., Magris, G. C., Patino, A., \& D’Alessio, P. 1992, Rev. Mex. Astron. Astrof., 24, 27

Calvet, N., Patino, A., Magris, G. C., \& D’Alessio, P. 1991, ApJ, 380, 617

Chandrasekhar, S. 1950/1960, Radiative Transfer (New York: Dover) Chiang, E. I., \& Goldreich, P. 1997, ApJ, 490, 368

Chiang, E. I., Joung, M. K., Creech-Eakman, M. J., et al. 2001, ApJ, 547, 1077

Cotera, A. S., Whitney, B. A., Young, E., et al. 2001, ApJ, 556, 958

D’Alessio, P., Calvet, N., \& Hartmann, L. 2001, ApJ, 553, 321

D’Alessio, P., Calvet, N., Hartmann, L., Lizano, S., \& Cantó, J. 1999, ApJ, 527, 893

D’Alessio, P., Canto, J., Calvet, N., \& Lizano, S. 1998, ApJ, 500, 411

Dominik, C., Dullemond, C. P., Waters, L. B. F. M., \& Walch, S. 2003 , A\&A, 398, 607

Draine, B. T., \& Lee, H. M. 1984, ApJ, 285, 89

Dullemond, C. P., Dominik, C., \& Natta, A. 2001, ApJ, 560, 957

Dullemond, C. P., van Zadelhoff, G. J., \& Natta, A. 2002, A\&A, 389, 464

Kenyon, S. J., \& Hartmann, L. 1987, ApJ, 323, 714

Kim, S., Martin, P. G., \& Hendry, P. D. 1994, ApJ, 422, 164

Malbet, F., \& Bertout, C. 1991, ApJ, 383, 814

Malbet, F., Lachaume, R., \& Monin, J.-L. 2001, A\&A, 379, 515

Mishchenko, M., Hovernier, J., \& Travis, L. (eds.) 2000, Light scattering by non-spherical particles (Academic Press)

Natta, A., Prusti, T., Neri, R., Wooden, D., \& Grinin, V. P. 2001, A\&A, 371, 186

Press, W., Teukolski, S., Vetterling, W., \& Flannery, B. 1992, Numerical Recipes in Fortran, Second edition (Cambridge University Press)

Strittmatter, P. A. 1974, A\&A, 32, 7

Testi, L., Natta, A., Shepherd, D. S., \& Wilner, D. J. 2001, ApJ, 554, 1087

Testi, L., Natta, A., Shepherd, D. S., \& Wilner, D. J. 2003, submitted van Zadelhoff, G.-J. Aikawa, Y., Hogerheijde, M. R., \& van Dishoeck, E. 2003, A\&A, 397, 789

Wolf, S., Padgett, D., \& Stapelfeldt, K. 2003, ApJ, 588, 373

Wood, K., Kenyon, S. J., Whitney, B., \& Turnbull, M. 1998, ApJ, 497, 404

Wood, K., Wolff, M. J., Bjorkman, J. E., \& Whitney, B. 2002, ApJ, 564,887 